\title{
Comparison of Small RNA Profiles of Glycine max and Glycine soja at Early Developmental Stages
}

\author{
Yuzhe Sun ${ }^{1,+}$, Zeta Mui ${ }^{2,3,+}$, Xuan Liu ${ }^{4,+}$, Aldrin Kay-Yuen Yim ${ }^{2,3}$, Hao Qin ${ }^{2}$, \\ Fuk-Ling Wong ${ }^{2,3}$, Ting-Fung Chan ${ }^{2,3}$, Siu-Ming Yiu 4 , Hon-Ming Lam 2,3,* \\ and Boon Leong $\operatorname{Lim}^{1,3, *}$ \\ 1 School of Biological Sciences, The University of Hong Kong, Pokfulam, Hong Kong, China; yzsun@hku.hk \\ 2 School of Life Sciences, The Chinese University of Hong Kong, Shatin, Hong Kong, China; \\ zeta.mui@gmail.com (Z.M.); aldrinlaurent@gmail.com (A.K.-Y.Y.); qinhao13@gmail.com (H.Q.); \\ wongfl@gmail.com (F.-L.W.); tf.chan@cuhk.edu.hk (T.-F.C.) \\ 3 Center for Soybean Research of the Partner State Key Laboratory of Agrobiotechnology, \\ The Chinese University of Hong Kong, Shatin, Hong Kong, China \\ 4 Department of Computer Science, The University of Hong Kong, Pokfulam, Hong Kong, China; \\ liuxuan@connect.hku.hk (X.L.); smyiu@cs.hku.hk (S.-M.Y.) \\ * Correspondence: honming@cuhk.edu.hk (H.-M.L.); bllim@hku.hk (B.L.L.); Tel.: +852-2299-0826 (B.L.L.) \\ + The authors contributed equally to this work.
}

Academic Editor: Marcello Iriti

Received: 28 October 2016; Accepted: 29 November 2016; Published: 6 December 2016

\begin{abstract}
Small RNAs, including microRNAs (miRNAs) and phased small interfering RNAs (phasiRNAs; from PHAS loci), play key roles in plant development. Cultivated soybean, Glycine max, contributes a great deal to food production, but, compared to its wild kin, Glycine soja, it may lose some genetic information during domestication. In this work, we analyzed the sRNA profiles of different tissues in both cultivated (C08) and wild soybeans (W05) at three stages of development. A total of 443 known miRNAs and 15 novel miRNAs showed varying abundances between different samples, but the miRNA profiles were generally similar in both accessions. Based on a sliding window analysis workflow that we developed, 50 PHAS loci generating 55 21-nucleotide phasiRNAs were identified in C08, and 46 phasiRNAs from 41 PHAS loci were identified in W05. In germinated seedlings, phasiRNAs were more abundant in C08 than in W05. Disease resistant TIR-NB-LRR genes constitute a very large family of PHAS loci. PhasiRNAs were also generated from several loci that encode for NAC transcription factors, Dicer-like 2 (DCL2), Pentatricopeptide Repeat (PPR), and Auxin Signaling F-box 3 (AFB3) proteins. To investigate the possible involvement of miRNAs in initiating the PHAS-phasiRNA pathway, miRNA target predictions were performed and 17 C08 miRNAs and 15 W05 miRNAs were predicted to trigger phasiRNAs biogenesis. In summary, we provide a comprehensive description of the sRNA profiles of wild versus cultivated soybeans, and discuss the possible roles of sRNAs during soybean germination.
\end{abstract}

Keywords: miRNA; phasiRNA; Glycine max; Glycine soja; sliding window analysis; PHAS loci; PHAS-phasiRNA pathway

\section{Introduction}

Small RNAs (sRNA) are important regulators of plant development and physiology. There are two main categories of sRNAs: Noncoding microRNAs (miRNA) and small interfering RNAs (siRNA) [1]. In plants, mature miRNAs are 20- to 22-nucleotide (nt) single-stranded RNAs that regulate target mRNA expression by cleavage or translational inhibition [2-4]. While most of the cleaved transcripts are degraded, some generate 21-nt secondary phased siRNAs [2,5]. In previous studies regarding these siRNAs, phased siRNAs that were not experimentally validated to be trans-acting 
were termed "phasiRNAs", while those found to be trans-acting were named "trans-acting siRNAs" (tasiRNAs) [6]. The loci that generate these phasiRNAs are called PHAS loci. These loci are also protein-coding, producing secondary cis-acting siRNAs that regulate themselves. In contrast, the loci that generate tasiRNAs are non-coding, and are termed Trans-Acting SiRNA (TAS) loci [6]. These TAS loci produce secondary siRNAs that target protein-coding genes found elsewhere in the genome. In Arabidopsis thaliana, some tasiRNAs are generated by the cleavage of the noncoding TAS transcripts (TAS1/2/3/4) [2,5,7], which act in trans in cleaving target mRNAs. Currently, at least four TAS loci have been identified in Arabidopsis. Argonaute 1 (AGO1)-miR173 initiates tasiRNA formation in TAS1 and TAS2 transcripts, and the generated tasiRNAs primarily target $P P R$ protein family transcripts, which are involved in RNA editing in chloroplasts and mitochondria [8-10]. TAS3 is triggered by miR390 and its phasiRNA has been experimentally proven to slow down a plant's transition from juvenile to adult by targeting auxin responsive factors (ARFs) [11]. TAS4 is triggered by miR828 and then one of its tasiRNAs targets, MYB113, regulates the anthocyanin production pathway [12]. Additionally, miR482-triggered TAS5 was found in tomatoes and dual miR156 and/or miR529-mediated TAS6 was found in Physcomitrella patens. Nonetheless, the production of phasiRNA/tasiRNA requires the involvement of miRNA: Some AGO1/7-bound miRNAs triggers the cleavage of PHAS or TAS mRNAs into $5^{\prime}$ and $3^{\prime}$ transcript fragments. Subsequently, multiple proteins participate in this process, including AGO1/7, suppressor of gene silencing 3 (SGS3), RNA-dependent RNA polymerase 6 (RDR6), and DCL proteins to form a series of phasiRNAs [13-16]. Either the $5^{\prime}$ or the $3^{\prime}$ fragments are then converted into double-stranded RNAs (dsRNAs) by RDR6 to form dsRNA precursors. Finally, DCL proteins will cleave the dsRNA precursor in a phased pattern, starting from the miRNA-guided cleavage site [6]. The cleaved fragments are oriented head to tail and all have precisely the same length. phasiRNAs repress the target mRNA by translational inhibition or by causing the degradation of target mRNAs [17-20].

Cultivated soybean (Glycine max) is an important crop for the production of edible oil and as a source of dietary protein $[21,22]$. As soybeans are strict cleistogamic pollinators, genomic variation may largely be reduced. The genetic homogeneity of this plant has been further intensified by cultivation. Wild soybeans (Glycine soja), on the other hand, may have retained some genetic traits that disappeared from the cultivated varieties after domestication. However, previous studies showed that the G. soja genome contains consensus sequences that represented a $97.65 \%$ coverage of G. max genome sequences [23]. However, the two soybean subspecies exhibit strong morphological diversity (seed color, seed size, leaf shape, etc.) and exhibit different responses to biotic and abiotic stress $[21,24]$. To date, hundreds of miRNAs and siRNAs have been identified on a genome-wide scale in soybeans $[21,25,26]$. In order to compare sRNA profiles between cultivated and wild soybeans, and to find potential sRNA transcriptional determinants that contributed to the phenotypic differences, we analyzed 14 small RNA libraries, created from different tissues across developmental stages from both cultivated soybeans (C08) and wild soybeans (W05). A strategy to identify PHAS loci and phasiRNA using a slide-window workflow was also developed. Using this method, differential phasiRNA profiles were observed in various developmental stages of both cultivated and wild soybeans.

\section{Results}

\subsection{Construction of Small RNA Libraries from Glycine max and Glycine soja}

Fourteen sRNA libraries were constructed from cultivated soybean accession C08 (Glycine max) and its wild progenitor W05 (Glycine soja), including roots and aerial parts. Root samples were collected from germinated seedlings (gr), young seedlings (yr), and seedlings (sr). Aerial part tissues, which included the cotyledons, were collected from germinated seedlings (ga), young seedlings (ya), and seedlings (sp). Additionally, trifoliate samples were collected from soybean seedlings (st) (Table S1).

Small RNA reads in 18 to 30 nucleotides (nt) range were retained. After removal of low quality and adaptor sequences, a total of 85,327,846 and 86,812,643 clean reads were yielded from C08 and W05 
libraries, respectively (Table S1). The reads were then mapped to either single or multiple positions in the genome. In total, $67,855,161$ and $63,974,900$ mapped reads $(79.5 \%$ and $73.7 \%$ of the total) were retained in C08 and W05, respectively, corresponding to 11,175,309 and 9,894,780 unique sequences (Table S1). The length distribution of mapped reads revealed that the population of small RNAs in different size clusters varied among different tissues (Figure S1). sRNAs 20-24 nt in size were the most abundant, which was consistent with a former report [27]. By comparing the 20-24 nt sRNA profiles, it was shown that W05 was particularly rich in $24 \mathrm{nt}$ sRNA (Figure S1).

\subsection{Identification of Novel miRNAs and Differential miRNA Expression in Glycine max and Glycine soja}

As only $2.35 \%$ of the total genome sequences are different between G. max and G. soja [23], the G. max genome (Wm82.a2.v1) was used as reference in miRNA identification and PHAS loci and phasiRNA prediction. Because gene location and annotation are different in these two genomes, it is difficult to integrate miRNA information with phasiRNA comparison. Although the mapping of G. soja libraries to the G. max genome leads to the loss of some sRNA information specific to G. soja, it helps to identify differential expression of sRNA between the two species.

A total of 621 known miRNAs (Table S2a) and 15 novel miRNAs (Table S3) were found. We also analyzed miRNA differential expression. In total, 443 known miRNAs and 15 novel miRNAs were found to be differentially accumulated in at least two pairwise comparisons (Tables S2b and S3).

Using psRNATarget, most of the novel miRNAs were predicted to either target transcription factors or development related proteins (Table S3). Embryo sac development arrest 14 (EDA 14) and F-box family proteins are strongly related to soybean development, especially seed germination. On the other hand, GRAS family transcription factor, targets of which were highly accumulated in young seedling roots and seedling roots, regulates plant development in root patterning [28]. Novel_miRNA_1, Novel_miRNA_9, and Novel_miRNA_15 targeted NAC transcription factors, which are responsive to biotic and abiotic stress responses [29]. Relative expressions of novel_miRNA_14 in 14 samples were further validated by qRT-PCR (Figure 1). The results were consistent between qRT-PCR and sRNA sequencing.
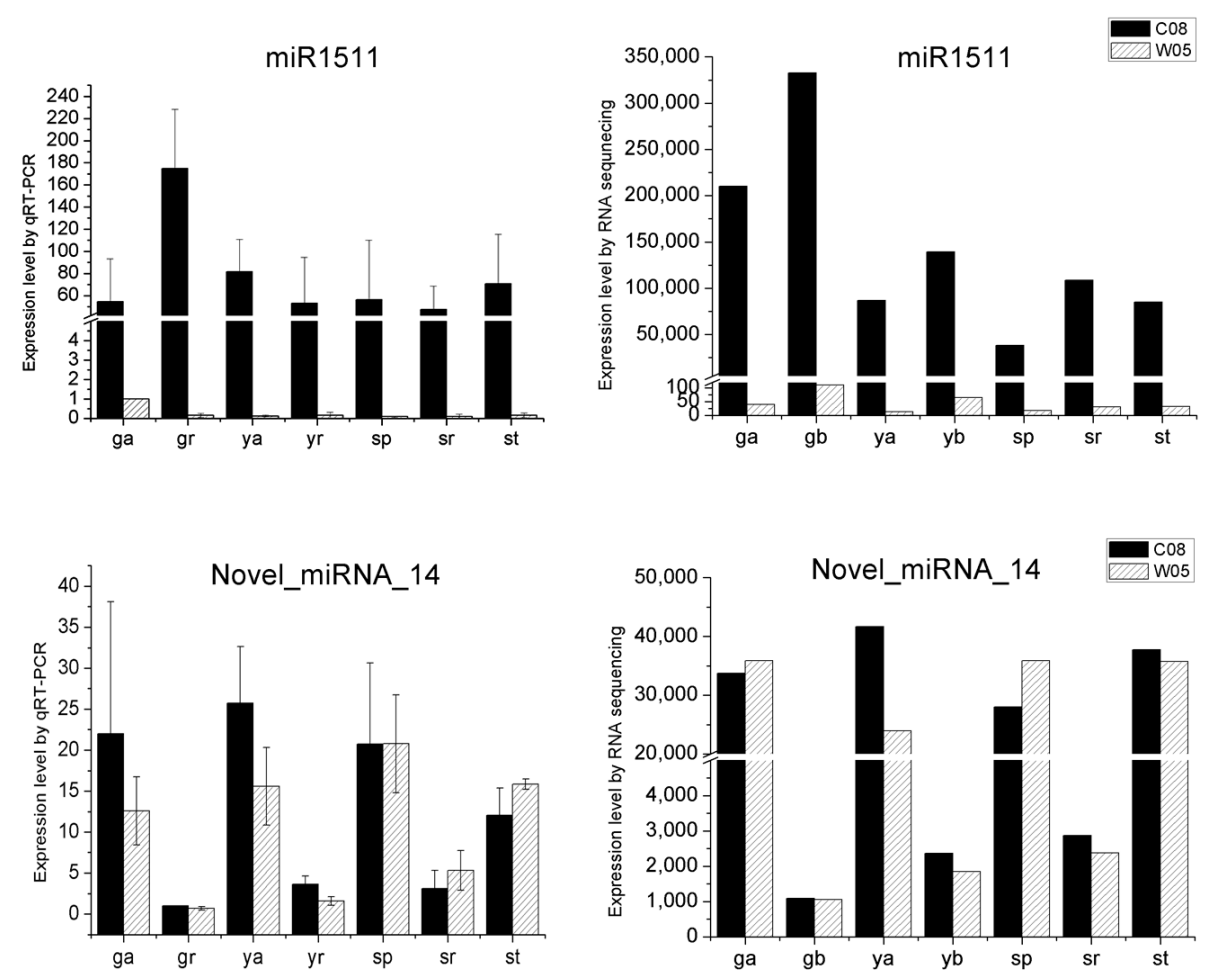

Figure 1. Cont. 

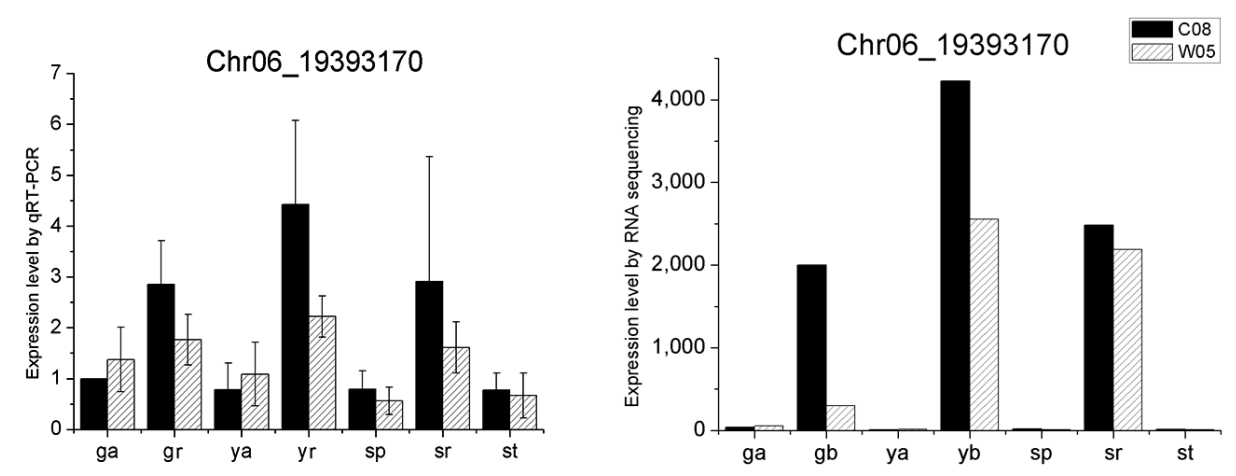

Figure 1. Validation of differential expressions of sRNAs. The differential expressions of miRNAs and phasiRNAs identified by sRNA sequencing (right) were validated by qRT-PCR (left). $Y$-axis showed the normalized reads of sRNA in 14 samples (right) and relative expression levels (left). Error bar shows the standard deviation (SD) of the three biological replicates used in qRT-PCR.

\subsection{Tissue Types and Developmental Stages Are Significant Determinants of miRNA Profiles for Both Wild and Cultivated Soybeans}

The correlation matrix showed that there are strong correlations among the roots of young seedlings and seedlings in both wild and cultivated soybeans, as indicated by the cluster of blue squares in the upper left corner (gwr vs. gcr) (Figure 2). A strong correlation was also found between the roots and the aerial parts of germinated seedlings of wild and cultivated soybeans (gwa vs. gca). In general, all seven C08 samples were positively correlated with the corresponding W05 samples of the same developmental stage or tissue type, which indicated that there were no fundamental differences in miRNA expression between C08 and W05. Two samples of W05, the trifoliate and primary leaves, showed substantial correlations (Figure 2).

To further investigate if there were significant differences between C08 and W05, we calculated the fold-change $\left(\log _{2}\right)$ in miRNA abundances in samples from W05 against corresponding samples from C08 (Figure S2). miRNAs with less than a two-fold difference (W05/C08) in abundance were removed. miRNAs in germinated seedlings were more diverse than those in other developmental stages, which implied that miRNA profile differences between C08 and W05 were largely in germinated seedlings. Particularly, miR1511 was dramatically enriched in all the tissues of C08, and, for miR156r, its abundance was higher in C08, in all samples except seedling trifoliates (swt_sct) (Figure S2).

\section{4. miRNA Profiles}

miRNAs with total reads $>3000$ reads in seven samples of C08 or W05 were studied (Figure 3, Table S4). Ten known miRNAs were differentially accumulated between C08 and W05 cotyledons (Figure 3, Table S4a) in which only miR1510a/b, miR1511, miR156r, and miR5670b had high counts. While miR1511 had 210,228 counts in C08 cotyledons, only 40 counts were found in W05, which indicated that mRNA regulation involving miR1511 had been initiated in C08 but not in W05. miR156r and miR5670 also had a much higher abundance in C08 cotyledons than in W05 cotyledons. By contrast, $\mathrm{miR} 1510 \mathrm{a} / \mathrm{b}$ abundance was more than seven-fold higher in W05 than in C08 (Table S4a). However, when we examined seven known miRNAs and Novel_miRNA_2 that were differentially accumulated between C08 and W05 germinating roots, the normalized absolute counts of miR156r and miR5670 were low (Table S4b). Nevertheless, miR1511 maintained high abundance in C08 roots like in C08 cotyledons, and the fold change (W05/C08) of miR1510b-5p was high (Table S4b). Twenty-two known miRNAs and two novel miRNAs in C08 were differentially accumulated between cotyledons and roots; 15 known miRNAs and two novel miRNAs in W05 were differentially accumulated between cotyledons and roots (Figure 3, Table S4c). Seventeen miRNAs in C08 were highly accumulated in leaves and seven in roots, while 10 miRNAs in W05 were highly accumulated in leaves and seven in 
roots (Table S4c). Two novel miRNAs, Novel_miRNA_11 and Novel_miRNA_14, were found in both C08 and W05 (Table S4c).

Changes in miRNA levels were also compared between developmental stages under our growth conditions. Six known miRNAs and Novel_miRNA_2 were commonly and differentially accumulated between the four germinated seedling samples and four young seedling samples (Figure 3, Table S4d). Additionally, only miR2109-5p had common and differential abundances between young seedling samples and seedling samples (Figure 3, Table S4e).

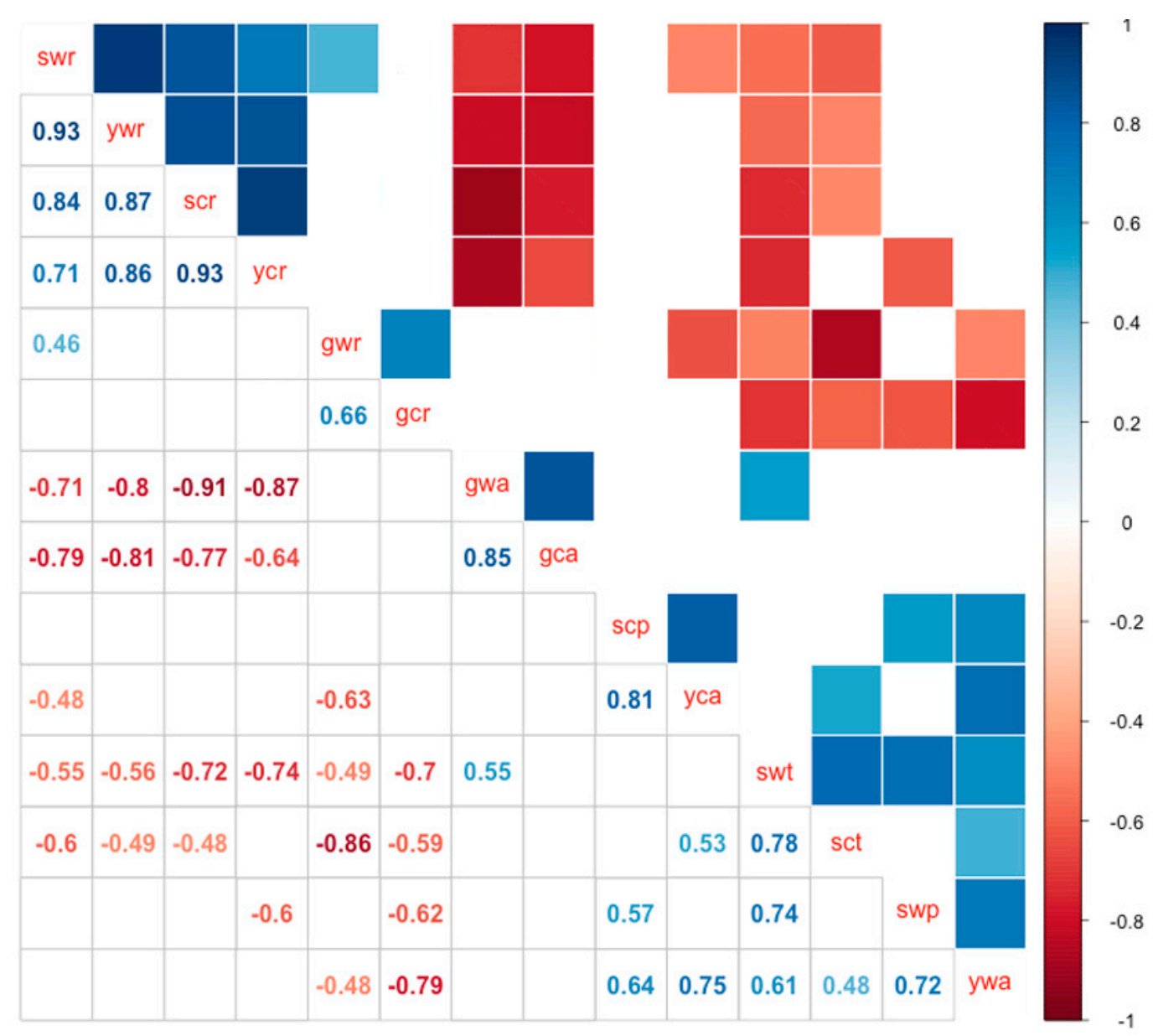

Figure 2. Pairwise correlation matrix of differentially-expressed miRNAs across tissue types, developmental stages, and wild and cultivated accessions. Pearson correlations between pairwise samples among all 14 experimental conditions were calculated by considering differentially-expressed miRNAs between each pairwise comparison as the distance between the two conditions. Blue boxes indicate positive correlation values $(0 \leq r \leq 1$; with 1 being complete positive correlation) and red boxes represent negative correlation values $(-1 \leq r \leq 0$, with -1 being complete anti-correlation) between samples. Correlation with $p$-values $>0.1$ are not shown in this matrix. The order of the rows was determined by the hierarchical clustering of the correlation matrix. Closely-related conditions, such as conditions with the same type of tissues, were therefore grouped together. "gca" and "gcr": aerial part and roots, respectively, of germinated seedlings of C08; "yca" and "ycr": aerial part and roots, respectively, of young seedlings of C08; "sct": trifoliates of C08 seedlings; "scp" and "scr": Primary leaves and roots, respectively, of C08; "gwa" and "gwr": aerial part and roots, respectively, of germinated seedlings of W05; "ywa" and "ywr": Aerial part and roots, respectively, of young seedlings of W05; "swt": Trifoliates of W05 seedlings; "swp" and "swr": Primary leaves and roots, respectively, of W05. 


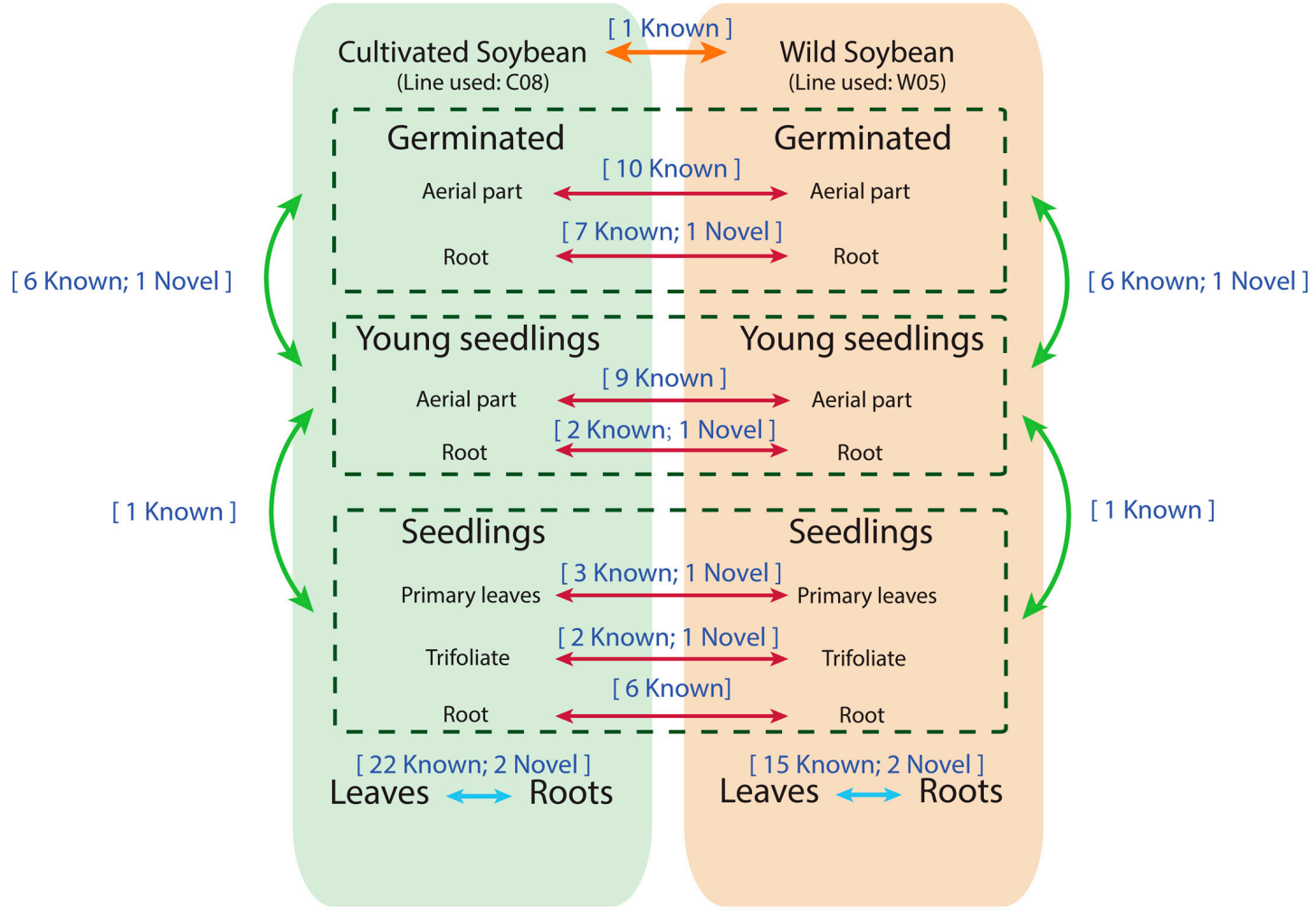

\section{$\leftrightarrow \leftrightarrow$ ) Any pairwise comparisons}

$\longleftrightarrow$ : Comparing all C08 tissues with all W05 tissues

$\longleftrightarrow$ : Comparing the same developmental stage between C08 \& W05 within the same tissue : Comparing among developmental stages within C08 or W05

: Comparing leaves (aerial parts/primary leaves/trifoliate) with roots within the same soybean line

[ \# miRNAs ] : Number of differentially expressed (absolute $\log _{2}$ fold change $\geq 2$ ) miRNA species of the indicated comparison

Figure 3. Differential miRNA expressions between different samples. We studied miRNAs with $>3000$ counts in seven samples of C08 or W05. Differential expression analysis was performed between C08 samples and W05 samples (orange and red arrows), between leaf samples and root samples (blue arrows), and between developmental stages (green arrows). Details of these miRNAs are presented in Table S4. 
Table 1. PHAS regions and their annotations in soybean.

\begin{tabular}{|c|c|c|c|c|c|c|}
\hline Chr & PHAS Start & PHAS End & Accession & Gene Name & Homologous Gene & Annotation \\
\hline Chr02 & $45,229,200$ & $45,229,892$ & $\mathrm{C} 08$ & NA & NA & NA \\
\hline Chr03 & $35,270,999$ & $35,271,544$ & $\mathrm{C} 08$ & Glyma.03G136600 & AT2G41750 & DTW domain-containing protein \\
\hline Chr04 & $47,362,862$ & $47,363,575$ & $\mathrm{C} 08$ & NA & NA & NA \\
\hline Chr04 & $49,035,971$ & $49,037,012$ & C08,W05 & Glyma.04G219600 & AT5G36930 & Disease resistance protein (TIR-NB-LRR class) family \\
\hline Chr05 & 656,827 & 657,414 & C08 & Glyma.05G006900 & AT3G14470 & NB-ARC domain-containing disease resistance protein \\
\hline Chr05 & $38,268,854$ & $38,269,817$ & C08,W05 & Glyma.05G198500 & AT5G23570 & SGS3, XS zinc finger domain-containing protein \\
\hline Chr06 & $11,932,996$ & $11,934,011$ & C08,W05 & Glyma.06G146200 & AT5G36930 & Disease resistance protein (TIR-NB-LRR class) family \\
\hline Chr06 & $19,392,730$ & $19,393,674$ & C08,W05 & Glyma.06G205100 & AT5G17680 & Disease resistance protein (TIR-NB-LRR class), putative \\
\hline Chr06 & $39,170,442$ & $39,171,407$ & W05 & Glyma.06G239000 & AT5G17680 & Disease resistance protein (TIR-NB-LRR class), putative \\
\hline Chr06 & $43,287,288$ & $43,287,977$ & C08,W05 & Glyma.06G254300 & AT3G14470 & NB-ARC domain-containing disease resistance protein \\
\hline Chr06 & $47,410,720$ & $47,411,412$ & C08,W05 & Glyma.06G285500 & AT5G17680 & Disease resistance protein (TIR-NB-LRR class), putative \\
\hline Chr07 & $4,028,350$ & $4,029,084$ & C08,W05 & Glyma.07G048000 & AT4G35580 & NTL9 NAC transcription factor-like 9 \\
\hline Chr08 & $41,923,481$ & $41,923,921$ & C08,W05 & Glyma.08G301200 & AT5G36930 & Disease resistance protein (TIR-NB-LRR class) family \\
\hline Chr08 & $41,923,963$ & $41,924,634$ & C08 & Glyma.08G301200 & AT5G36930 & Disease resistance protein (TIR-NB-LRR class) family \\
\hline Chr09 & $2,041,836$ & $2,042,507$ & C08,W05 & Glyma.09G025300 & AT3G03300 & ATDCL2,DCL2 dicer-like 2 \\
\hline Chr09 & $2,737,112$ & $2,737,899$ & C08,W05 & Glyma.09G032400 & NA & NA \\
\hline Chr09 & $47,565,020$ & $47,565,481$ & W05 & Glyma.09G256600 & AT3G22470 & Pentatricopeptide repeat (PPR) superfamily protein \\
\hline Chr11 & $30,572,116$ & $30,573,147$ & C08,W05 & Glyma.11G212800 & AT3G14460 & LRR and NB-ARC domains-containing disease resistance protein \\
\hline Chr12 & $31,231,341$ & $31,232,533$ & C08,W05 & Glyma.12G163000 & AT1G31540 & Disease resistance protein (TIR-NB-LRR class) family \\
\hline Chr12 & $39,530,806$ & $39,531,225$ & $\mathrm{C} 08$ & Glyma.12G236500 & AT4G27220 & NB-ARC domain-containing disease resistance protein \\
\hline Chr13 & $19,268,532$ & $19,269,329$ & $\mathrm{C} 08$ & NA & NA & NA \\
\hline Chr13 & $29,861,566$ & $29,862,427$ & C08 & Glyma.13G184800 & AT3G14460 & LRR and NB-ARC domains-containing disease resistance protein \\
\hline Chr13 & $29,875,305$ & $29,876,627$ & C08,W05 & Glyma.13G184900 & AT3G14470 & NB-ARC domain-containing disease resistance protein \\
\hline Chr13 & $30,174,985$ & $30,175,782$ & C08,W05 & Glyma.13G187900 & AT3G14470 & NB-ARC domain-containing disease resistance protein \\
\hline Chr13 & $30,207,736$ & $30,208,554$ & C08,W05 & Glyma.13G188300 & AT3G14470 & NB-ARC domain-containing disease resistance protein \\
\hline Chr13 & $30,389,026$ & $30,390,362$ & C08,W05 & Glyma.13G190300 & AT3G14470 & NB-ARC domain-containing disease resistance protein \\
\hline Chr13 & $30,664,791$ & $30,665,882$ & C08 & Glyma.13G193300 & AT3G14470 & NB-ARC domain-containing disease resistance protein \\
\hline Chr13 & $30,727,236$ & $30,728,889$ & C08,W05 & Glyma.13G194100 & AT3G14470 & NB-ARC domain-containing disease resistance protein \\
\hline Chr13 & $30,915,467$ & $30,916,705$ & C08,W05 & Glyma.13G195600 & AT3G14460 & LRR and NB-ARC domains-containing disease resistance protein \\
\hline Chr14 & $3,878,256$ & $3,878,843$ & C08 & NA & NA & NA \\
\hline Chr15 & $43,699,964$ & $43,700,551$ & C08,W05 & Glyma.15G232400 & AT5G41540 & Disease resistance protein (TIR-NB-LRR class) family \\
\hline Chr15 & $43,746,080$ & $43,746,499$ & C08 & Glyma.15G232800 & AT3G14460 & LRR and NB-ARC domains-containing disease resistance protein \\
\hline Chr15 & $44,546,466$ & $44,547,735$ & C08,W05 & NA & NA & NA \\
\hline Chr16 & $1,452,507$ & $1,453,157$ & C08,W05 & Glyma.16G016600 & AT4G35580 & NTL9 NAC transcription factor-like 9 \\
\hline Chr16 & $1,453,270$ & $1,453,905$ & C08 & Glyma.16G016600 & AT4G35580 & NTL9 NAC transcription factor-like 9 \\
\hline Chr16 & $1,461,742$ & $1,462,416$ & C08,W05 & Glyma.16G016700 & AT4G35580 & NTL9 NAC transcription factor-like 9 \\
\hline Chr16 & $1,462,506$ & $1,463,177$ & C08 & Glyma.16G016700 & AT4G35580 & NTL9 NAC transcription factor-like 9 \\
\hline
\end{tabular}


Table 1. Cont

\begin{tabular}{|c|c|c|c|c|c|c|}
\hline Chr & PHAS Start & PHAS End & Accession & Gene Name & Homologous Gene & Annotation \\
\hline Chr16 & $2,941,895$ & $2,942,482$ & C08,W05 & Glyma.16G031100 & AT2G46100 & Nuclear transport factor 2 (NTF2) family protein \\
\hline Chr16 & $4,855,988$ & $4,856,743$ & C08,W05 & Glyma.16G050500 & AT1G12820 & AFB3 auxin signaling F-box 3 protein \\
\hline Chr16 & $5,770,754$ & $5,771,618$ & C08,W05 & Glyma.16G058900 & NA & NA \\
\hline Chr16 & $10,648,349$ & $10,648,999$ & C08,W05 & Glyma.16G087100 & AT5G36930 & Disease resistance protein (TIR-NB-LRR class) family \\
\hline Chr16 & $30,784,351$ & $30,785,128$ & C08,W05 & Glyma.16G147100 & NA & NA \\
\hline Chr16 & $32,109,362$ & $32,110,117$ & C08,W05 & Glyma.16G161900 & AT1G12775 & Pentatricopeptide repeat (PPR) superfamily protein \\
\hline Chr16 & $32,167,989$ & $32,168,613$ & C08 & Glyma.16G162800 & AT1G62670 & RPF2 RNA processing factor 2 \\
\hline Chr16 & $32,182,422$ & $32,182,883$ & $\mathrm{C} 08$ & Glyma.16G163100 & AT1G12700 & ATP binding;nucleic acid binding;helicases \\
\hline Chr16 & $32,453,347$ & $32,453,892$ & W05 & Glyma.16G165400 & AT1G12300 & Tetratricopeptide repeat (TPR)-like superfamily protein \\
\hline Chr16 & $33,378,642$ & $33,379,535$ & W05 & Glyma.16G173200 & NA & 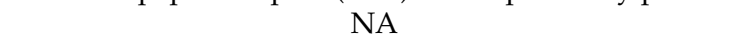 \\
\hline Chr16 & $35,681,818$ & $35,682,321$ & W05 & Glyma.16G194800 & AT1G63130 & Tetratricopeptide repeat (TPR)-like superfamily protein \\
\hline Chr16 & $35,687,005$ & $35,687,634$ & C08,W05 & Glyma.16G195000 & AT1G12775 & Pentatricopeptide repeat (PPR) superfamily protein \\
\hline Chr16 & $35,692,915$ & $35,693,523$ & C08,W05 & Glyma.16G195100 & AT1G12700 & ATP binding;nucleic acid binding;helicases \\
\hline Chr16 & $35,883,305$ & $35,883,850$ & W05 & Glyma.16G197600 & AT1G62910 & Pentatricopeptide repeat (PPR) superfamily protein \\
\hline Chr16 & $36,088,255$ & $36,088,695$ & C08,W05 & Glyma.16G199700 & AT1G62930 & Tetratricopeptide repeat (TPR)-like superfamily protein \\
\hline Chr16 & $36,970,009$ & $36,970,575$ & C08 & Glyma.16G210600 & AT5G36930 & Disease resistance protein (TIR-NB-LRR class) family \\
\hline Chr17 & $40,464,414$ & $40,465,001$ & C08,W05 & Glyma.17G249500 & NA & NA \\
\hline Chr19 & $34,719,617$ & $34,720,288$ & C08,W05 & Glyma.19G100200 & AT1G12820 & AFB3 auxin signaling F-box 3 protein \\
\hline Chr19 & $40,040,875$ & $40,041,548$ & C08,W05 & NA & NA & NA \\
\hline Chr20 & $31,854,969$ & $31,855,514$ & W05 & NA & NA & NA \\
\hline
\end{tabular}




\subsection{Genome-Wide Identification of PHAS Loci and PhasiRNAs}

Twenty-one-nucleotide phasiRNAs, triggered by miRNAs, are a typical feature of phasiRNA initiation [30]. In this study, a pipeline has been developed for genome-wide identification of the PHAS loci, phasiRNAs, and miRNA-phasiRNA pathways. The pipeline started with high-throughput next-generation RNA sequencing data that then went through multiple filtering stages (Figure 4). Using this sliding window method, 50 and 41 PHAS loci were predicted in C08 and W05, respectively, at a stringent threshold $p$ value of $<1 \times 10^{-6}$ (Table 1, Figure 4). After mapping, all the phasiRNAs with a total count $>3000$ in the seven samples of each variety were retained. Seventy-one and 55 phasiRNAs were identified in C08 and W05 libraries, respectively. Phase graphs were then plotted for each locus, and those loci with more than two phases of phasiRNAs were discarded (Figures S3 and S4). Finally, 55 phasiRNAs 21-nt in length from C08 and 46 phasiRNAs 21-nt in length from W05 libraries, met the above criteria (Table S5).

A

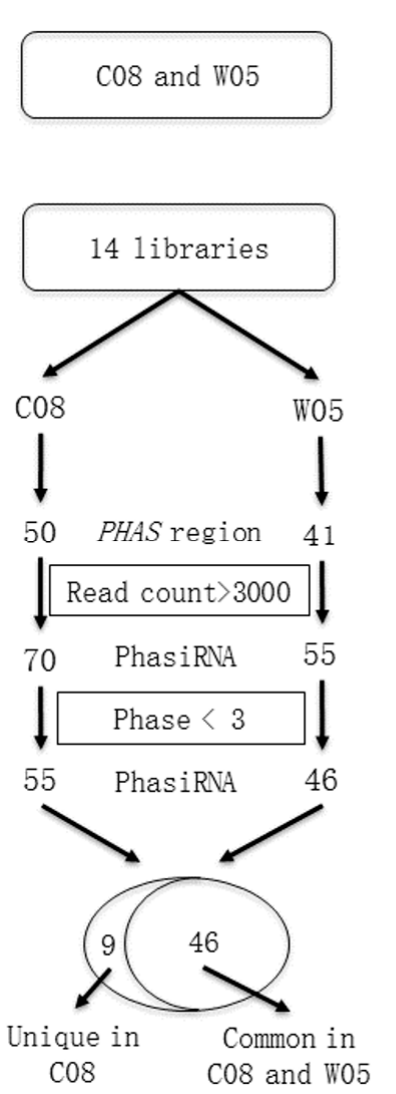

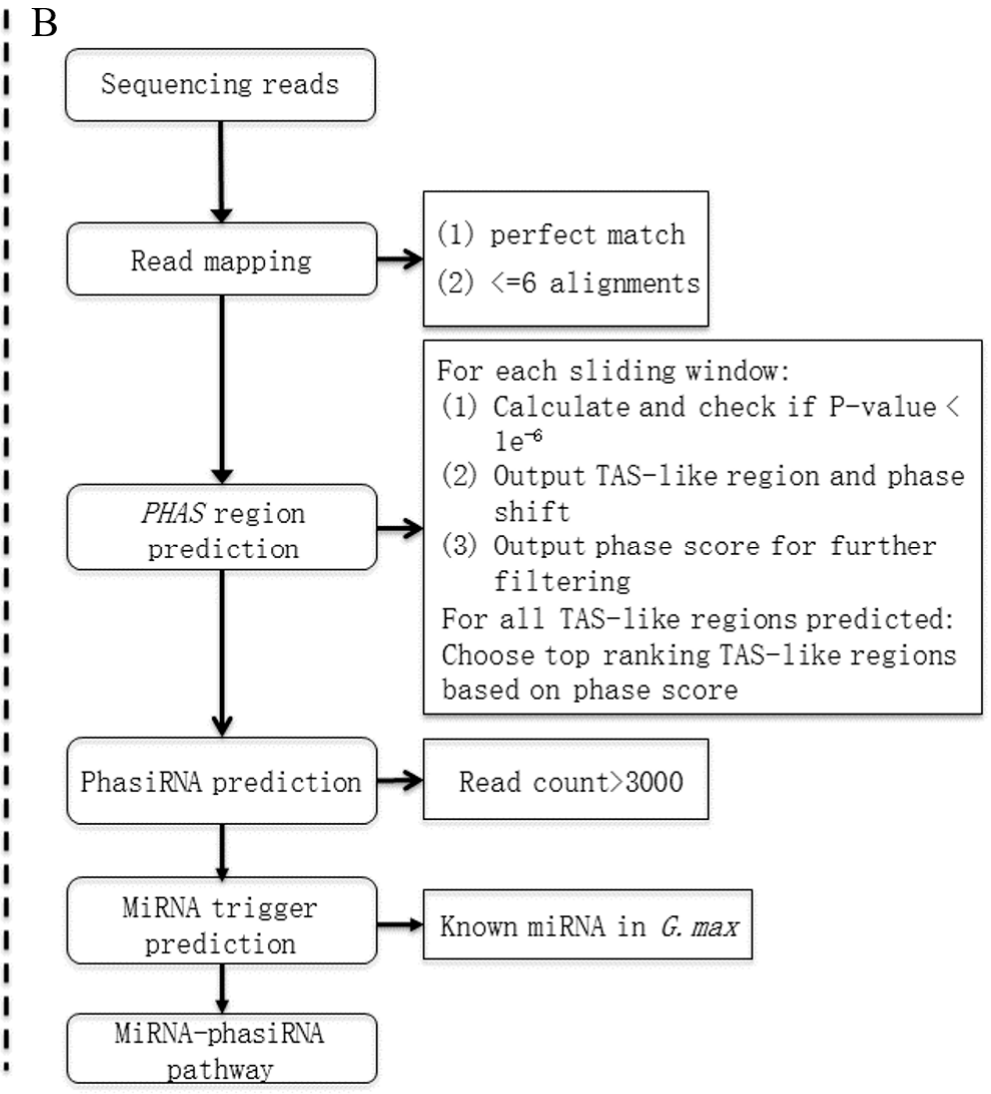

Figure 4. The pipeline for phasiRNA predictions: (A) The workflow for the comparisons between the phasiRNA profiles of $\mathrm{C} 08$ and W05. The numbers of common and unique phasiRNAs are shown in the Venn diagram; (B) The workflow for phasiRNA identification. Sequencing reads were mapped to the reference genome Gmax_275_Wm82.a2.v1 and only those reads that could be mapped to at most six positions on the reference genome with perfect matches were used. A sliding-window method was then applied to predict genome-wide PHAS regions using the pattern of mapped reads based on the ranking of $p$-values and phase scores. In each predicted PHAS region, loci with on-phase high-count reads (generating phasiRNAs $>3000$ total reads in seven sample of each variety) were selected and regarded as putative phasiRNA-generating loci. A characterization of each putative phasiRNA was performed to identify any possible miRNA triggers that initiated the biogenesis of phasiRNAs. The final product was a proposed pathway for each miRNA-phasiRNA pair. 
Considering that phasiRNAs are triggered by miRNA, target prediction of miRNAs was performed. A total of 5716 binding sites were predicted. Binding sites that were within $420 \mathrm{nt}$ $(20 \times 21 \mathrm{nt})$ of the phasiRNAs were retained. Fifty-two phasiRNAs in C08 were predicted to be triggered by 17 miRNAs and 50 phasiRNAs in W05 were predicted to be triggered by 15 miRNAs (Table S6a). Fourteen miRNAs were predicted to dually trigger the biogenesis of phasiRNAs in C08 and W05. miR2118, miR390, and miR9761 were only found in C08, and miR1536 could only trigger phasiRNAs in W05 (Table S6a). Furthermore, four miRNAs (miR1507, miR1509, miR1510, and miR1514) in C08 and five miRNAs (miR1508, miR1510, miR1514, miR393, and miR5674) in W05 were predicted to trigger more than one PHAS locus. Interestingly, miR1510 can target multiple PHAS loci, including three regions in C08 and four regions in W05 (Table S6b and Table S6c). The abundances of three sRNAs, miR1511, novel_miRNA_14, and Chr06_19393170, were validated by qRT-PCR in 14 soybean samples (Figure 1). Their expression patterns corresponded closely with the RNA sequencing data.

\subsection{Differential Expression of PhasiRNA in Different Tissues}

A number of phasiRNAs were differentially or preferentially accumulated in some tissues (Table 2). In germinated seedling roots, most of the phasiRNAs had lower reads in W05 than in C08, except the phasiRNAs from Glyma.13G187900, Glyma.07G048000, and Glyma.16G147100. Similarly, in seedling trifoliates, most of the phasiRNAs had lower reads in W05 than in C08 except phasiRNAs from the two PHAS genes (Table 2). In all seven tissues, eight phasiRNAs from Glyma.13G187900 and Glyma.07G048000, had higher abundance in W05. Furthermore, one phasiRNA from AFB3 had higher reads in the cotyledons of W05 (Table 2).

Dramatic differences in phasiRNA abundances could be seen between the aerial parts and roots across all three development stages of both accessions. The phasiRNAs shown in green were preferentially accumulated in leaves and the phasiRNAs shown in red were preferentially accumulated in roots (Table 3). The preferences were independent of developmental stages and accessions. Interestingly, three TIR-NB-LRR loci (Glyma.04G219600, Glyma.06G285500, and Glyma.12G163000) that produce phasiRNAs were preferentially accumulated in leaves, whereas another TIR-NB-LRR locus (Glyma.06G205100) that produces phasiRNAs was accumulated almost exclusively in roots (Table 3).

In addition, four phasiRNAs from Chr15_44546856_44547257 and five phasiRNAs from Glyma.09G032400 showed higher abundances in aerial parts at all three stages (Table 3). A phasiRNA generated from a PPR locus is highly accumulated in leaves at all three stages (Table 3). PPR proteins participate in post-transcriptional processes including RNA editing, which occurs in plastids and mitochondria [10,31]. Leaf cells have a large number of chloroplasts while roots cells only have plastids. Hence, the higher abundance of this phasiRNA in leaves may be required to control the higher PPR transcript abundances in the leaves. However, one phasiRNA from an AFB3 locus (Glyma.16G050500) had elevated abundance in roots (Table 3).

Additionally, nine phasiRNAs were exclusively found in C08 (Table S5d). These nine phasiRNAs belonged to a TIR-NB-LRR gene, a NTL9 (NAC domain-containing protein 9-like) gene, a LRR and NB-ARC domain, and five other un-annotated genes (Table S5d). Abundances of two phasiRNAs from the NTL9 gene gradually increased over the three developmental stages in both leaves and roots. Chr04_47363101 preferentially enriched in aerial tissues while Chr06_19393086 from a TIR-NB-LRR gene accumulated in roots (Table S5d). 
Table 2. Changes in the abundances of phasiRNAs in W05 (wild) and C08 (cultivated) soybeans.

\begin{tabular}{|c|c|c|c|c|c|c|c|c|c|}
\hline \multirow{2}{*}{ Gene Name } & \multirow{2}{*}{ Annotation } & \multirow{2}{*}{$\begin{array}{l}\text { Number of } \\
\text { phasiRNAs }\end{array}$} & \multicolumn{7}{|c|}{ Fold Change of Expression Level (W05/C08) } \\
\hline & & & $\mathrm{Ga}$ & Gr & Ya & Yr & Sp & $\mathrm{Sr}$ & St \\
\hline Glyma.04G219600 & Disease resistance protein (TIR-NB-LRR class) & 4 & 1.1 & 0.4 & 0.6 & 1.1 & 0.7 & 1.2 & 0.5 \\
\hline Glyma.06G146200 & Disease resistance protein (TIR-NB-LRR class) & 4 & 1.4 & 0.4 & 0.9 & 1.1 & 0.3 & 0.6 & 0.3 \\
\hline Glyma.06G205100 & Disease resistance protein (TIR-NB-LRR class) & 2 & 1.1 & 0.3 & 1.4 & 0.8 & 1.0 & 1.5 & 0.4 \\
\hline Glyma.06G285500 & Disease resistance protein (TIR-NB-LRR class) & 1 & 1.8 & 0.5 & 1.6 & 1.5 & 1.5 & 1.2 & 1.1 \\
\hline Glyma.08G301200 & Disease resistance protein (TIR-NB-LRR class) & 1 & 0.9 & 0.5 & 0.5 & 0.7 & 0.6 & 1.0 & 0.4 \\
\hline Glyma.12G163000 & Disease resistance protein (TIR-NB-LRR class) & 2 & 0.6 & 0.5 & 0.9 & 1.1 & 1.3 & 1.1 & 0.6 \\
\hline Glyma.13G187900 & NB-ARC domain-containing disease resistance protein & 4 & 3.6 & 2.2 & 2.5 & 2.2 & 1.5 & 2.3 & 3.5 \\
\hline Glyma.11G212800 & LRR and NB-ARC domains-containing disease resistance protein & 1 & 0.6 & 0.5 & 0.6 & 1.0 & 0.8 & 1.5 & 0.5 \\
\hline Glyma.07G048000 & NTL9 NAC transcription factor-like 9 & 4 & 2.0 & 1.9 & 1.8 & 2.5 & 1.6 & 3.2 & 1.7 \\
\hline Glyma.16G016600 & NTL9 NAC transcription factor-like 9 & 1 & 1.1 & 0.3 & 0.4 & 1.0 & 0.3 & 1.1 & 0.3 \\
\hline Glyma.09G025300 & ATDCL2, DCL2 & 2 & 1.0 & 0.3 & 0.9 & 1.2 & 0.6 & 1.4 & 1.0 \\
\hline Glyma.16G050500 & AFB3 auxin signaling F-box 3 & 1 & 2.7 & 0.9 & 1.0 & 0.8 & 0.5 & 0.7 & 1.3 \\
\hline Glyma.16G161900 & Pentatricopeptide repeat (PPR) superfamily protein & 1 & 1.0 & 0.5 & 0.8 & 1.0 & 0.6 & 1.2 & 0.7 \\
\hline Glyma.16G058900 & NA & 3 & 1.0 & 0.4 & 0.9 & 1.0 & 0.6 & 1.1 & 0.5 \\
\hline Glyma.16G147100 & NA & 3 & 0.9 & 1.4 & 0.6 & 1.0 & 0.4 & 1.1 & 0.6 \\
\hline Chr15_44546856_44547257 & NA & 4 & 0.6 & 0.2 & 0.6 & 0.8 & 0.4 & 0.9 & 0.6 \\
\hline Glyma.09G032400 & NA & 5 & 1.1 & 0.5 & 0.7 & 0.8 & 0.7 & 1.0 & 0.5 \\
\hline
\end{tabular}

Ga: Germinated seedling aerial parts. Gr: Germinated seedling roots. Ya: Young seedling aerial parts. Yr: Young seedling roots. Sp: Seedling primary leaves. Sr: Seedling roots. St: Seedling trifoliates. Red: Fold change $\leq 0.5$ (higher abundance in C08). Green: Fold change $\geq 2$ (higher abundance in W05). 
Table 3. Differential amounts of phasiRNAs in the PHAS regions in leaves versus roots.

\begin{tabular}{|c|c|c|c|c|c|c|c|c|c|c|}
\hline \multirow[t]{2}{*}{ Gene Name } & \multirow[t]{2}{*}{ Annotation } & \multirow{2}{*}{$\begin{array}{l}\text { Number of } \\
\text { phasiRNA }\end{array}$} & \multicolumn{2}{|c|}{$\begin{array}{c}\text { Leaf/Root } \\
\text { (Germinated Seedlings) }\end{array}$} & \multicolumn{2}{|c|}{$\begin{array}{c}\text { Leaf/Root } \\
\text { (Young Seedlings) }\end{array}$} & \multicolumn{2}{|c|}{$\begin{array}{l}\text { Leaf/Root } \\
\text { (Seedlings) }\end{array}$} & \multicolumn{2}{|c|}{$\begin{array}{c}\text { Leaf/Root } \\
\text { (Three Stages) }\end{array}$} \\
\hline & & & $\mathrm{C} 08$ & W05 & $\mathrm{C} 08$ & W05 & $\mathrm{C} 08$ & W05 & $\mathrm{C} 08$ & W05 \\
\hline Glyma.04G219600 & Disease resistance protein (TIR-NB-LRR class) & 4 & 2.2 & 5.4 & 2.6 & 1.6 & 5.3 & 3.9 & 2.9 & 3.0 \\
\hline Glyma.06G146200 & Disease resistance protein (TIR-NB-LRR class) & 4 & 0.8 & 1.8 & 1.2 & 1.0 & 2.9 & 1.2 & 1.4 & 1.3 \\
\hline Glyma.06G205100 & Disease resistance protein (TIR-NB-LRR class) & 2 & 0.04 & 0.13 & 0.01 & 0.01 & 0.02 & 0.02 & 0.03 & 0.04 \\
\hline Glyma.06G285500 & Disease resistance protein (TIR-NB-LRR class) & 1 & 7.1 & 27.9 & 6.1 & 6.8 & 10.6 & 13.1 & 7.3 & 12.0 \\
\hline Glyma.08G301200 & Disease resistance protein (TIR-NB-LRR class) & 1 & 0.1 & 0.3 & 0.6 & 0.4 & 1.5 & 1.1 & 0.5 & 0.5 \\
\hline Glyma.12G163000 & Disease resistance protein (TIR-NB-LRR class) & 2 & 19.9 & 34.2 & 7.0 & 6.9 & 12.6 & 15.6 & 11.1 & 11.2 \\
\hline Glyma.13G187900 & NB-ARC domain-containing disease resistance protein & 4 & 1.1 & 1.4 & 1.4 & 1.6 & 4.8 & 2.7 & 2.2 & 1.9 \\
\hline Glyma.11G212800 & LRR and NB-ARC domains-containing disease resistance protein & 1 & 1.2 & 1.3 & 0.7 & 0.4 & 1.7 & 1.0 & 1.2 & 0.8 \\
\hline Glyma.07G048000 & NTL9 NAC transcription factor-like 9 & 4 & 1.3 & 1.2 & 0.4 & 0.3 & 1.0 & 0.6 & 0.8 & 0.6 \\
\hline Glyma.16G016600 & NTL9 NAC transcription factor-like 9 & 1 & 1.0 & 3.2 & 1.2 & 0.6 & 2.5 & 0.7 & 1.5 & 1.2 \\
\hline Glyma.09G025300 & ATDCL2, DCL2 & 2 & 0.4 & 1.4 & 0.6 & 0.5 & 1.4 & 0.6 & 0.7 & 0.7 \\
\hline Glyma.16G050500 & AFB3 auxin signaling F-box 3 & 1 & 0.1 & 0.3 & 0.3 & 0.4 & 0.7 & 0.5 & 0.5 & 0.4 \\
\hline Glyma.16G161900 & Pentatricopeptide repeat (PPR) superfamily protein & 1 & 3.4 & 7.5 & 2.7 & 2.3 & 6.8 & 3.1 & 3.9 & 3.9 \\
\hline Glyma.16G058900 & NA & 3 & 0.8 & 1.6 & 1.1 & 0.9 & 2.3 & 1.4 & 1.1 & 1.3 \\
\hline Glyma.16G147100 & NA & 3 & 0.4 & 0.9 & 1.0 & 0.5 & 1.6 & 0.8 & 0.8 & 0.7 \\
\hline Chr15_44546856_44547257 & NA & 4 & 1.3 & 3.8 & 3.0 & 2.2 & 5.8 & 2.6 & 2.4 & 2.9 \\
\hline Glyma.09G032400 & NA & 5 & 1.3 & 2.0 & 3.1 & 2.7 & 4.4 & 2.8 & 2.5 & 2.5 \\
\hline
\end{tabular}

Red: Fold change $\leq 0.5$ (higher abundance in the roots); Green: Fold change $\geq 2$ (high abundance in the leaves). 


\section{Discussion}

\subsection{Differential Expressions of sRNAs between Cultivated and Wild Soybean and Their Biological Significance}

The sRNA network is an important mechanism that regulates gene expression at transcriptional and post-transcriptional levels. In this study, we focused on the analyses of miRNAs and phasiRNAs in two soybean accessions including seven tissues from three developmental stages. During seed germination, cotyledon is a major tissue in the aerial part of the plant and is the first photosynthetic structure to develop from the hypocotyl. Although a large number of miRNAs were found to be differentially expressed between two individual samples, much fewer miRNAs were universally differentially expressed between accessions (C08 vs. W05), tissues (root vs. leaf) and developmental stages (germinated seedling vs. young seedling vs. seedling). Only miR1511 exhibited significantly different abundances across all seven tissues between the two accessions, implying that the miRNA profiles are similar between C08 and W05 (Table S4f). The abundance of miR1511 was greatly elevated in C08 (3276 times higher than that in W05) during germination (Table S4f). miR1511 has been studied in G. max (C08), and G. soja (W05) [32,33], in the nodules of Medicago [34], and in Phaseolus vulgaris [35]. In soybeans, glutamine amidotransferase class-I, which is an important enzyme involved in glutamine biosynthesis, was identified as the target of miR1511 [33]. Here, the abundance of miR1511 was, by orders of magnitude, higher in all the tissue types of C08 than in W05, and its level was twice as high in the roots as in the leaves. Thus, we speculated that miR1511 might be an important factor in modulating nitrogen metabolism in the roots of C08 (Table S4f).

During plant development, the miRNA expression profile changes according to regulatory demand. Several pathways were strongly involved in plant juvenile-to-adult and adult-to-flowering phase transitions. One of the pathways, the age pathway, is mainly dictated by miRNAs. Two key miRNAs, miR156, and miR172, modulate juvenility and flowering. Previous studies showed that miR156 is necessary for maintaining juvenility in plants. The accumulation level of miR156 went down when the plant transitioned into adulthood and later to flowering. At the same time, abundance of miR172 gradually increases when plants enter the flowering (reproductive) phase, acting downstream of the miR156 targets, SPLs (Squamosa Promoter-Binding Protein-Likes). Higher miR172 abundance promotes juvenile-to-adult phase changes and flowering [36-40]. In our data set, miR156 was highly expressed in the cotyledons as well as roots of both C08 and W05, then the abundance declined in the leaves of young seedlings by almost 10 times (Table S4g). However, in C08 seedling leaves, miR156 kept on declining while, in W05 seedling leaves, the abundance retained. Meanwhile, miR172 expression increased dramatically in the young seedling leaves of both C08 and W05 compared to germinated seedling cotyledons, which was consistent with previous reports [41]. However, in the roots of W05, miR172 expression was stable in germinated seedlings and young seedlings, but declined in seedlings. This same decline was also observed in the roots of C08, starting in the young seedling stage, one stage earlier than in W05 (Table S4g). The decline of miR156 abundance and the elevation of miR172 abundance in the leaves of young seedlings could be a signal that suggests the initiation of phase transition. If so, the decline of miR172 abundance in roots happened earlier in C08 than in W05, suggesting that C08 may reach the reproductive growth sooner than W05.

\subsection{PHAS Loci and phasiRNAs in Soybean}

Secondary phasiRNAs are generated from PHAS loci. The annotation of the PHAS loci in this study showed that 23 out of $50(46 \%)$ in C08 and 17 out of $41(41.5 \%)$ in W05 were TIR-NB-LRR disease resistance coding genes (Table 1). The NB-LRR class of plant disease resistance (R) proteins has been shown to play an important role in defense signaling [42]. A large number of phasiRNAs were generated from NB-LRR loci in Medicago and Solanaceae, and the production of these phasiRNAs is probably related to plant defense mechanisms [5,43]. Additionally, our miRNA data showed that miR168, of which the target is AGO1, was differentially expressed between tissues and between accessions (Table S2b). In addition, two PHAS genes were annotated as SGS3 and 
DCL2 (Table 1). In Arabidopsis thaliana, AGO1, SGS3, and DCL2 are key proteins in the phasiRNA biosynthesis pathway [44]. phasiRNAs generated from these loci may therefore affect secondary phasiRNA production.

Compared to the same tissue from C08, the abundances of four phasiRNAs from NTL9 genes, three phasiRNAs from genes encoding proteins with the NB-ARC domain and 1 phasiRNA from the AFB3 gene were elevated in W05 cotyledons (Table 2). The overexpression of NAC factors in Arabidopsis and rice was shown to improve tolerance to biotic and abiotic stress [45]. W05 also exhibited strong salt tolerance in the early growth period [46]. Moreover, the $A F B 3$ protein is an auxin receptor, and in Arabidopsis, AFB3 coordinates primary and lateral root growth and the NAC4 transcription factor acts downstream of $A F B 3$ to control root nitrate responses [47]. Seeing as the AFB3 protein acts as a crucial receptor in the auxin-signaling pathway, which affects root elongation, it is reasonable to observe a higher abundance of this phasiRNA in roots. These possible links gave us hints that phasiRNAs might be related to multiple stress responses, while W05 happened to have strong salt tolerance in the early growth period [46]. Whether these elevated phasiRNAs are related to W05's responses to salt stress requires further investigation.

The two soybean lines possess different seed sizes and seed coat colors (Figure S5). Seeds of C08 are yellow and are larger than seeds of W05, which are black [21,46]. Large seed size was likely a trait actively selected for over the course of human domestication, and the yellow seed coat color may have been a by-product of this artificial selection. C08 cotyledons are larger in size, but their salt tolerance is much lower than W05 [46]. Larger, heavier seeds contain more nutrients, providing more energy for germination. In phasiRNA profiles, a great number of phasiRNAs were suppressed in W05 germinated roots (Table 2). These phenotypic differences may cause the differences in gene expressions and sRNA profiles between the two accessions during germination.

Interestingly, in both wild and cultivated accessions, three phasiRNA-producing TIR-NB-LRR loci (Glyma.04G219600, Glyma.06G285500, and Glyma.12G163000) were preferentially accumulated in leaves and a different TIR-NB-LRR phasiRNA-producing locus (Glyma.06G205100) was preferentially accumulated in roots, across all three developmental stages (Table 3). Considering roots and leaves face different pathogens in their respective environments, these TIR-NB-LRR loci originated, differentially accumulated phasiRNAs may play important roles in immune defense [48,49].

\section{3. miRNA-phasiRNA Regulation in Soybean}

Fifty-two phasiRNAs triggered by 17 miRNAs were identified in accession C08, and 50 phasiRNAs triggered by 15 miRNAs were identified in accession W05 (Table S5a). miR2118, miR390, and miR9761 were unique in C08, and miR1536 was unique in W05. We found the miR2118-induced phasiRNA (Chr03_35271156) was highly accumulated in C08. This secondary small RNA has a high sequence similarity to TIR-NBS-LRR mRNAs. In Medicago truncatula, some TIR-NBS-LRR disease resistance genes were validated as the targets of miR2118 [50]. It is possible that Chr03_35271156 phasiRNAs cleave or inhibit the translation of TIR-NBS-LRR mRNAs in order to simultaneously regulate multiple members of the same gene family. For other large plant gene families like PPR, this gene regulation role undertaken by phasiRNA has already been demonstrated [2,51]. In Arabidopsis thaliana, TAS3-derived tasiARFs, which are trans-acting small interfering RNAs triggered by miR390, target ARFs and regulate plant growth [52]. miR390 accumulated in both accessions but the corresponding phasiRNAs were only generated in C08. These phasiRNAs may play a role in the development of C08. In general, phasiRNAs can act as amplifiers that downregulate multiple highly homologous target genes.

We applied next-generation sequencing and analyzed 14 small RNA libraries generated from various tissues and developmental stages of wild and cultivated soybean. With these data, we found 458 (443 known and 15 novel) miRNAs to be differentially expressed. Additionally, a statistically rigorous pipeline to identify PHAS loci in soybean was developed based on previous work [6], which identified 50 PHAS loci containing 55 phasiRNAs in C08 and 41 PHAS loci containing 46 phasiRNAs in W05 (Figure 4). miRNAs that might trigger the biogenesis of phasiRNAs were also predicted (Table S5). 
This study provides a global view of sRNAs in C08 and W05. It can be a reference for future functional studies of sRNAs in Glycine max and Glycine soja.

\section{Materials and Methods}

\subsection{Plant Materials}

The phylogenetic relationship between C08 and W05 was reported previously [21]. C08 is a cultivated soybean from the USA, and is a close relative of Williams 82. W05 is a wild soybean originating from Henan Province, China.

Seed preparation. Since the seed coat of W05 seed is hard, the W05 seeds were scarified by a razor blade before germination to ensure a uniform germination rate. Germinated seedlings, young seedlings, and seedlings samples were collected, and their descriptions are as follows. Germinated seedling. C08 and scarified W05 seeds were germinated in 70\% tap-water-moistened vermiculite in the dark with a lid. After $72 \mathrm{~h}$, the lid was removed and germinated seedlings continued to grow. Four- to five-day-old seedlings were collected as germinated seedlings. Young seedlings. Seven-day-old seedlings from germination were then transferred to a hydroponic system with Hoagland's solution. Young seedling samples were collected when the primary leaves were fully opened. Seedlings. Seedlings samples were collected when the first set of trifoliate leaves were fully opened. Aerial part, trifoliate and root samples of seedling samples were collected.

\subsection{RNA Extraction and Sequencing of Small RNA}

Total RNA from soybean plants was extracted as previously described [53]. The total RNA was sent to the Beijing Genomics Institute (BGI) (Shenzhen, China). Fourteen small RNA libraries from different developmental stages and tissue types of wild and cultivated soybeans were sequenced using Illumina HiSeq ${ }^{\mathrm{TM}} 2000$ high throughput sequencing technology, as previously described, in order to obtain single-end, directional reads [51].

\subsection{Reads Pre-Processing and Mapping for Downstream Analyses}

As only $2.35 \%$ of the $937.5-\mathrm{Mb}$ genome sequences differ between G. max and G. soja [23], high quality G. max genome sequences were used for mapping. After adaptor-trimming, reads were mapped against the soybean genome (Wm82.a2.v1) with different methods for sRNA discovery and expression tabulation purposes. To consider all possible new miRNA/phasiRNA loci, two aligners, Bowtie version 0.12.7 [54] (with -v 0 -k 20 -best -strata -S option) and Soap3-dp [55] were used to identify reads without mismatches and were mapped to either single or multiple positions in the genome, accepting up to 20 valid alignments. For expression tabulation purposes, reads were mapped with Bowtie version 0.12 .7 (with $-\mathrm{v} 0-\mathrm{k} 1$-best option), accepting uniquely mapped reads, as well as one valid alignment per read for multi-mapped reads. The mapping results are summarized in Supplemental Table S1.

\subsection{Generation of rRNA, $t R N A$, Repeat Regions, snRNA/snoRNA Annotations}

Ribosomal RNA, tRNA, and repeat sequences in the reference genome were annotated by RNAmmer 1.2 [56], tRNAscan-SE 1.23 [57] and RepeatMasker 4.0.5 [58], respectively. Covariance models (CMs) of snRNAs/snoRNAs were extracted from Rfam (12.0) CM [59] using cmfetch of Infernal-1.1 [60], and was used to search against the Wm82.a2.v1 reference genome. Hits with $E$-values below the inclusion threshold of 0.01 were accepted as snRNAs/snoRNAs.

\subsection{Soybean MicroRNA Discovery and Annotation}

The mapped reads for discovery purposes were fed into the miRNA prediction software miR-PREFeR [61]. Predicted miRNA precursors and their corresponding mature miRNAs were annotated by BLASTn [62] against the miRNA precursors in miRBase (release 21) [63], and the best 
miRBase hit with query coverage $\geq 90 \%$ and identity $\geq 90 \%$ over the length of the matched sequence was considered the same miRNA precursor. Those remaining were considered to be novel miRNA candidates. Predicted miRNAs arising from rRNA, tRNA, repeat regions, snRNA, and snoRNA loci were discarded.

The genomic locations of all known soybean miRNA precursors on soybean genome v1.0 (genome-build accession: NCBI Assembly: GCA_000004515.1) were obtained from miRBase. To determine the location of known miRNAs on the new reference genome assembly Wm82.a2.v1, miRNAs with a 500-bp flanking region on each side were extracted for BLASTn against the reference genome Wm82.a2.v1. Hits with $100 \%$ identity and $100 \%$ coverage over the flanking regions were accepted as the location of the extracted region in the reference genome, and precursors without a match with these criteria were subjected to the next round of mapping. The process was repeated with 200-bp flanking lengths, 50-bp flanking lengths, and, finally, no flanking regions to determine the genomic location of miRNA genes in the genome assembly Wm82.a2.v1.

\subsection{Normalization and Differential Gene Expression Calling of miRNAs}

To tabulate miRNA expression levels, read counts were tabulated from the expression tabulation purpose mapped reads. The size factor used to normalize the read counts was calculated with the DESeq package [64], and the normalized read counts were then analyzed by the DEGseq package [65] to identify differentially expressed miRNAs. Pairwise library comparisons for each $M I R$ gene were performed and MIR genes that had a $p$-value $<0.001$ and an absolute log fold-change $\geq 2$ indicated a differential expression between the two conditions being compared.

\subsection{Target Prediction of miRNAs}

The targets of miRNAs were predicted with psRNATarget [66] using the Wm82.a2.v1 transcripts as a database. The targets predicted with an expectation value $\leq 3$ were considered to be confident predictions.

\subsection{Prediction of phasiRNA Regions}

Reads in the discovery purpose-mapped read set with no more than six mapped positions in the genome were selected for feeding into the phasiRNA prediction algorithm. Tailored for deep sequencing data, a sliding window method was adopted to detect PHAS regions, where phasiRNAs were generated based on read mapping patterns [30,67]. In References [30,67], the $231 \mathrm{nt}$ downstream window of each $5^{\prime}$ mapping position was examined and a $p$-values test was performed in the window. The number of windows to be examined equals to the number of read mapping positions on genome, and it was positively correlated with sequencing depth. We assumed that the phasiRNA was likely to be $21 \mathrm{nt}$ long and reduced the window size to be 10 times the length (i.e., $210 \mathrm{nt}$ ) of the phasiRNA being tested. Starting from the $5^{\prime}$ position of each read mapped region on genome, we started $p$-values tests $[30,67]$ in the $210 \mathrm{nt}$ window and the window was shifted $s(s=21)$ positions at each step towards the $3^{\prime}$ end of the reference genome after each test. Therefore, the number of windows to be examined equals to the total length of read mapped regions divided by 21 and would not increase as sequencing depth deepened. Phase shift ( $x$, from 1 to 21) was defined to indicate the position of the phasiRNAs generated from one PHAS region relative to the window start position. Thus, different PHAS regions predicted from the same window could be distinguished from one another. For each window and each phase shift $x$, a $p$-values test was applied to all mapped reads. If the $p$-value was below $1 \times 10^{-6}$, it implied that on-phase $(x)$ s-long reads were significantly more abundant than those that were not on-phase $(x)$. Thus, a PHAS region was called within the window on phase $x$. After all PHAS regions were called, we further selected the top 50 highest-ranking candidates, based on phase scores [18]. 


\subsection{Quantitative RT-PCR}

Quantitative reverse transcription polymerase chain reaction (qRT-PCR) analysis was carried out using cDNA samples on a StepOnePlus Real-Time PCR System (Applied Biosystems, Waltham, CA, USA). cDNAs were synthesized from $4 \mu \mathrm{g}$ of total RNA by the miRNA First Strand Synthesis Kit (Clontech, CA, USA), and SYBR Green Master Mix (Applied Biosystems) was used in the qPCR experiments. miR1520d was used as the internal control. For every transcript, each cDNA sample was analyzed in triplicate, and the relative transcript abundance was calculated by normalizing to the maximum concentration. The assessment of accumulation comparing different targets was determined by the $2^{-\Delta \Delta C t}$ method.

Supplementary Materials: Supplementary materials can be found at www.mdpi.com/1422-0067/17/12/2043/s1.

Acknowledgments: This work is supported by grants from Hong Kong Research Grants Council (Collaborative Research Fund: CUHK3/CRF/11G; Area of Excellence: AoE/M-05/12), Lo Kwee-Seong Biomedical Research Fund, and the Innovation and Technology Fund (Funding Support to Partner State Key Laboratories in Hong Kong) of the HKSAR, China. Jee-Yan Chu copy-edited the manuscript. We thank John Richards for proofreading the manuscript.

Author Contributions: Zeta Mui, Aldrin Kay-Yuen Yim and Hao Qin performed differential expression analyses and annotations on the miRNAs. Xuan Liu, Yuzhe Sun and Siu-Ming Yiu developed the computational workflow for the discovery of the phasiRNAs. Xuan Liu and Yuzhe Sun performed miRNA-phasiRNA predictions. Fuk-Ling Wong prepared the samples for sRNA sequencing and qPCR validation. Yuzhe Sun performed validations of sRNAs. Ting-Fung Chan performed annotations on the miRNAs and proofread the manuscript. Hon-Ming Lam designed the experimental setup. Yuzhe Sun, Zeta Mui, Hon-Ming Lam and Boon Leong Lim co-wrote the manuscript.

Conflicts of Interest: The authors declare no conflicts of interest.

\section{References}

1. Khraiwesh, B.; Zhu, J.K.; Zhu, J. Role of miRNAs and siRNAs in biotic and abiotic stress responses of plants. Biochim. Biophys. Acta 2012, 1819, 137-148. [CrossRef] [PubMed]

2. Xia, R.; Meyers, B.C.; Liu, Z.; Beers, E.P.; Ye, S.; Liu, Z. MicroRNA superfamilies descended from miR390 and their roles in secondary small interfering RNA biogenesis in eudicots. Plant Cell 2013, 25, 1555-1572. [CrossRef] [PubMed]

3. Reinhart, B.J.; Weinstein, E.G.; Rhoades, M.W.; Bartel, B.; Bartel, D.P. MicroRNAs in plants. Genes Dev. 2002, 16, 1616-1626. [CrossRef] [PubMed]

4. Khraiwesh, B.; Arif, M.A.; Seumel, G.I.; Ossowski, S.; Weigel, D.; Reski, R.; Frank, W. Transcriptional control of gene expression by microRNAs. Cell 2010, 140, 111-122. [CrossRef] [PubMed]

5. Zhai, J.; Jeong, D.H.; de Paoli, E.; Park, S.; Rosen, B.D.; Li, Y.; Gonzalez, A.J.; Yan, Z.; Kitto, S.L.; Grusak, M.A.; et al. MicroRNAs as master regulators of the plant NB-LRR defense gene family via the production of phased, trans-acting siRNAs. Genes Dev. 2011, 25, 2540-2553. [CrossRef] [PubMed]

6. Fei, Q.; Xia, R.; Meyers, B.C. Phased, secondary, small interfering RNAs in posttranscriptional regulatory networks. Plant Cell 2013, 25, 2400-2415. [CrossRef] [PubMed]

7. Yoshikawa, M.; Peragine, A.; Park, M.Y.; Poethig, R.S. A pathway for the biogenesis of trans-acting siRNAs in Arabidopsis. Genes Dev. 2005, 19, 2164-2175. [CrossRef] [PubMed]

8. Allen, E.; Xie, Z.; Gustafson, A.M.; Carrington, J.C. MicroRNA-directed phasing during trans-acting siRNA biogenesis in plants. Cell 2005, 121, 207-221. [CrossRef] [PubMed]

9. Felippes, F.F.; Weigel, D. Triggering the formation of tasiRNAs in Arabidopsis thaliana: The role of microRNA miR173. EMBO Rep. 2009, 10, 264-270. [CrossRef] [PubMed]

10. Cheng, S.; Gutmann, B.; Zhong, X.; Ye, Y.; Fisher, M.F.; Bai, F.; Castleden, I.; Song, Y.; Song, B.; Huang, J.; et al. Redefining the structural motifs that determine RNA binding and RNA editing by pentatricopeptide repeat proteins in land plants. Plant J. 2016. [CrossRef] [PubMed]

11. Montgomery, T.A.; Howell, M.D.; Cuperus, J.T.; Li, D.; Hansen, J.E.; Alexander, A.L.; Chapman, E.J.; Fahlgren, N.; Allen, E.; Carrington, J.C. Specificity of ARGONAUTE7-miR390 interaction and dual functionality in TAS3 trans-acting siRNA formation. Cell 2008, 133, 128-141. [CrossRef] [PubMed] 
12. Luo, Q.J.; Mittal, A.; Jia, F.; Rock, C.D. An autoregulatory feedback loop involving PAP1 and TAS4 in response to sugars in Arabidopsis. Plant Mol. Biol. 2012, 80, 117-129. [CrossRef] [PubMed]

13. Peragine, A.; Yoshikawa, M.; Wu, G.; Albrecht, H.L.; Poethig, R.S. SGS3 and SGS2/SDE1/RDR6 are required for juvenile development and the production of trans-acting siRNAs in Arabidopsis. Genes Dev. 2004, 18, 2368-2379. [CrossRef] [PubMed]

14. Vazquez, F.; Vaucheret, H.; Rajagopalan, R.; Lepers, C.; Gasciolli, V.; Mallory, A.C.; Hilbert, J.L.; Bartel, D.P.; Crete, P. Endogenous trans-acting siRNAs regulate the accumulation of Arabidopsis mRNAs. Mol. Cell 2004, 16, 69-79. [CrossRef] [PubMed]

15. Adenot, X.; Elmayan, T.; Lauressergues, D.; Boutet, S.; Bouche, N.; Gasciolli, V.; Vaucheret, H. DRB4-dependent TAS3 trans-acting siRNAs control leaf morphology through AGO7. Curr. Biol. 2006, 16, 927-932. [CrossRef] [PubMed]

16. Montgomery, T.A.; Yoo, S.J.; Fahlgren, N.; Gilbert, S.D.; Howell, M.D.; Sullivan, C.M.; Alexander, A.; Nguyen, G.; Allen, E.; Ahn, J.H.; et al. AGO1-mir173 complex initiates phased siRNA formation in plants. Proc. Natl. Acad. Sci. USA 2008, 105, 20055-20062. [CrossRef] [PubMed]

17. Allen, E.; Howell, M.D. miRNAs in the biogenesis of trans-acting siRNAs in higher plants. Semin. Cell Dev. Biol. 2010, 21, 798-804. [CrossRef] [PubMed]

18. Howell, M.D.; Fahlgren, N.; Chapman, E.J.; Cumbie, J.S.; Sullivan, C.M.; Givan, S.A.; Kasschau, K.D.; Carrington, J.C. Genome-wide analysis of the RNA-DEPENDENT RNA POLYMERASE6/DICER-LIKE4 pathway in Arabidopsis reveals dependency on miRNA- and tasiRNA-directed targeting. Plant Cell 2007, 19, 926-942. [CrossRef] [PubMed]

19. Rajagopalan, R.; Vaucheret, H.; Trejo, J.; Bartel, D.P. A diverse and evolutionarily fluid set of microRNAs in Arabidopsis thaliana. Genes Dev. 2006, 20, 3407-3425. [CrossRef] [PubMed]

20. Parent, J.S.; Bouteiller, N.; Elmayan, T.; Vaucheret, H. Respective contributions of Arabidopsis DCL2 and DCL4 to RNA silencing. Plant J. 2015, 81, 223-232. [CrossRef] [PubMed]

21. Lam, H.M.; Xu, X.; Liu, X.; Chen, W.; Yang, G.; Wong, F.L.; Li, M.W.; He, W.; Qin, N.; Wang, B.; et al. Resequencing of 31 wild and cultivated soybean genomes identifies patterns of genetic diversity and selection. Nat. Genet. 2010, 42, 1053-1059. [CrossRef] [PubMed]

22. Schmutz, J.; Cannon, S.B.; Schlueter, J.; Ma, J.; Mitros, T.; Nelson, W.; Hyten, D.L.; Song, Q.; Thelen, J.J.; Cheng, J.; et al. Genome sequence of the palaeopolyploid soybean. Nature 2010, 463, 178-183. [CrossRef] [PubMed]

23. Kim, M.Y.; Lee, S.; Van, K.; Kim, T.H.; Jeong, S.C.; Choi, I.Y.; Kim, D.S.; Lee, Y.S.; Park, D.; Ma, J.; et al. Whole-genome sequencing and intensive analysis of the undomesticated soybean (Glycine soja Sieb. and Zucc.) genome. Proc. Natl. Acad. Sci. USA 2010, 107, 22032-22037. [CrossRef] [PubMed]

24. Li, W.Y.; Shao, G.; Lam, H.M. Ectopic expression of GmPAP3 alleviates oxidative damage caused by salinity and osmotic stresses. New Phytol. 2008, 178, 80-91. [CrossRef] [PubMed]

25. Song, Q.X.; Liu, Y.F.; Hu, X.Y.; Zhang, W.K.; Ma, B.; Chen, S.Y.; Zhang, J.S. Identification of miRNAs and their target genes in developing soybean seeds by deep sequencing. BMC Plant Biol. 2011, 11, 5. [CrossRef] [PubMed]

26. Arikit, S.; Xia, R.; Kakrana, A.; Huang, K.; Zhai, J.; Yan, Z.; Valdes-Lopez, O.; Prince, S.; Musket, T.A.; Nguyen, H.T.; et al. An atlas of soybean small RNAs identifies phased siRNAs from hundreds of coding genes. Plant Cell 2014, 26, 4584-4601. [CrossRef] [PubMed]

27. Joshi, T.; Yan, Z.; Libault, M.; Jeong, D.H.; Park, S.; Green, P.J.; Sherrier, D.J.; Farmer, A.; May, G.; Meyers, B.C.; et al. Prediction of novel miRNAs and associated target genes in Glycine max. BMC Bioinform. 2010, 11 (Suppl. 1), S14. [CrossRef] [PubMed]

28. Hirsch, S.; Oldroyd, G.E. GRAS-domain transcription factors that regulate plant development. Plant Signal. Behav. 2009, 4, 698-700. [CrossRef] [PubMed]

29. Nuruzzaman, M.; Sharoni, A.M.; Kikuchi, S. Roles of NAC transcription factors in the regulation of biotic and abiotic stress responses in plants. Front. Microbiol. 2013, 4, 248. [CrossRef] [PubMed]

30. Chen, H.M.; Li, Y.H.; Wu, S.H. Bioinformatic prediction and experimental validation of a microRNA-directed tandem trans-acting siRNA cascade in Arabidopsis. Proc. Natl. Acad. Sci. USA 2007, 104, 3318-3323. [CrossRef] [PubMed]

31. Barkan, A.; Small, I. Pentatricopeptide repeat proteins in plants. Annu. Rev. Plant Biol. 2014, 65, 415-442. [CrossRef] [PubMed] 
32. Htwe, N.M.; Luo, Z.Q.; Jin, L.G.; Nadon, B.; Wang, K.J.; Qiu, L.J. Functional marker development of miR1511-InDel and allelic diversity within the genus Glycine. BMC Genom. 2015, 16, 467. [CrossRef] [PubMed]

33. Dong, Z.; Shi, L.; Wang, Y.; Chen, L.; Cai, Z.; Jin, J.; Li, X. Identification and dynamic regulation of microRNAs involved in salt stress responses in functional soybean nodules by high-throughput sequencing. Int. J. Mol. Sci. 2013, 14, 2717-2738. [CrossRef] [PubMed]

34. Lelandais-Briere, C.; Naya, L.; Sallet, E.; Calenge, F.; Frugier, F.; Hartmann, C.; Gouzy, J.; Crespi, M. Genome-wide Medicago truncatula small RNA analysis revealed novel microRNAs and isoforms differentially regulated in roots and nodules. Plant Cell 2009, 21, 2780-2796. [CrossRef] [PubMed]

35. Pelaez, P.; Trejo, M.S.; Iniguez, L.P.; Estrada-Navarrete, G.; Covarrubias, A.A.; Reyes, J.L.; Sanchez, F. Identification and characterization of microRNAs in Phaseolus vulgaris by high-throughput sequencing. BMC Genom. 2012, 13, 83. [CrossRef] [PubMed]

36. Wu, G.; Park, M.Y.; Conway, S.R.; Wang, J.W.; Weigel, D.; Poethig, R.S. The sequential action of miR156 and miR172 regulates developmental timing in Arabidopsis. Cell 2009, 138, 750-759. [CrossRef] [PubMed]

37. Teotia, S.; Tang, G. To bloom or not to bloom: Role of microRNAs in plant flowering. Mol. Plant 2015, 8, 359-377. [CrossRef] [PubMed]

38. Wu, G.; Poethig, R.S. Temporal regulation of shoot development in Arabidopsis thaliana by miR156 and its target SPL3. Development 2006, 133, 3539-3547. [CrossRef] [PubMed]

39. Gandikota, M.; Birkenbihl, R.P.; Hohmann, S.; Cardon, G.H.; Saedler, H.; Huijser, P. The miRNA156/157 recognition element in the 3' UTR of the Arabidopsis SBP box gene SPL3 prevents early flowering by translational inhibition in seedlings. Plant J. 2007, 49, 683-693. [CrossRef] [PubMed]

40. Jung, J.H.; Ju, Y.; Seo, P.J.; Lee, J.H.; Park, C.M. The SOC1-SPL module integrates photoperiod and gibberellic acid signals to control flowering time in Arabidopsis. Plant J. 2012, 69, 577-588. [CrossRef] [PubMed]

41. Lauter, N.; Kampani, A.; Carlson, S.; Goebel, M.; Moose, S.P. MicroRNA172 down-regulates glossy15 to promote vegetative phase change in maize. Proc. Natl. Acad. Sci. USA 2005, 102, 9412-9417. [CrossRef] [PubMed]

42. Wan, L.; Zhang, X.; Williams, S.J.; Ve, T.; Bernoux, M.; Sohn, K.H.; Jones, J.D.; Dodds, P.N.; Kobe, B. Crystallization and preliminary X-ray diffraction analyses of the tir domains of three TIR-NB-LRR proteins that are involved in disease resistance in Arabidopsis thaliana. Acta Crystallogr. Sect. F Struct. Biol. Cryst. Commun. 2013, 69, 1275-1280. [CrossRef] [PubMed]

43. Li, F.; Pignatta, D.; Bendix, C.; Brunkard, J.O.; Cohn, M.M.; Tung, J.; Sun, H.; Kumar, P.; Baker, B. MicroRNA regulation of plant innate immune receptors. Proc. Natl. Acad. Sci. USA 2012, 109, 1790-1795. [CrossRef] [PubMed]

44. Yoshikawa, M.; Iki, T.; Tsutsui, Y.; Miyashita, K.; Poethig, R.S.; Habu, Y.; Ishikawa, M. $3^{\prime}$ fragment of miR173-programmed RISC-cleaved RNA is protected from degradation in a complex with RISC and SGS3. Proc. Natl. Acad. Sci. USA 2013, 110, 4117-4122. [CrossRef] [PubMed]

45. Jensen, M.K.; Hagedorn, P.H.; de Torres-Zabala, M.; Grant, M.R.; Rung, J.H.; Collinge, D.B.; Lyngkjaer, M.F. Transcriptional regulation by an NAC (NAM-ATAF1,2-CUC2) transcription factor attenuates ABA signalling for efficient basal defence towards Blumeria graminis f. sp. hordei in Arabidopsis. Plant J. 2008, 56, 867-880. [PubMed]

46. Qi, X.; Li, M.W.; Xie, M.; Liu, X.; Ni, M.; Shao, G.; Song, C.; Kay-Yuen Yim, A.; Tao, Y.; Wong, F.L.; et al. Identification of a novel salt tolerance gene in wild soybean by whole-genome sequencing. Nat. Commun. 2014, 5, 4340. [CrossRef] [PubMed]

47. Vidal, E.A.; Araus, V.; Lu, C.; Parry, G.; Green, P.J.; Coruzzi, G.M.; Gutierrez, R.A. Nitrate-responsive miR393/AFB3 regulatory module controls root system architecture in Arabidopsis thaliana. Proc. Natl. Acad. Sci. USA 2010, 107, 4477-4482. [CrossRef] [PubMed]

48. Andika, I.B.; Maruyama, K.; Sun, L.; Kondo, H.; Tamada, T.; Suzuki, N. Differential contributions of plant dicer-like proteins to antiviral defences against potato virus $\times$ in leaves and roots. Plant J. 2015, 81, 781-793. [CrossRef] [PubMed]

49. Andika, I.B.; Kondo, H.; Tamada, T. Evidence that RNA silencing-mediated resistance to beet necrotic yellow vein virus is less effective in roots than in leaves. Mol. Plant Microbe Interact. 2005, 18, 194-204. [CrossRef] [PubMed] 
50. Jagadeeswaran, G.; Zheng, Y.; Li, Y.F.; Shukla, L.I.; Matts, J.; Hoyt, P.; Macmil, S.L.; Wiley, G.B.; Roe, B.A.; Zhang, W.; et al. Cloning and characterization of small RNAs from Medicago truncatula reveals four novel legume-specific microRNA families. New Phytol. 2009, 184, 85-98. [CrossRef] [PubMed]

51. Liang, C.; Liu, X.; Sun, Y.; Yiu, S.M.; Lim, B.L. Global small RNA analysis in fast-growing Arabidopsis thaliana with elevated concentrations of ATP and sugars. BMC Genom. 2014, 15, 116. [CrossRef] [PubMed]

52. Marin, E.; Jouannet, V.; Herz, A.; Lokerse, A.S.; Weijers, D.; Vaucheret, H.; Nussaume, L.; Crespi, M.D.; Maizel, A. miR390, Arabidopsis TAS3 tasiRNAs, and their AUXIN RESPONSE FACTOR targets define an autoregulatory network quantitatively regulating lateral root growth. Plant Cell 2010, 22, 1104-1117. [CrossRef] [PubMed]

53. Aushubel, F.; Brent, R.; Kingston, R.; Moore, D.; Seidman, J.; Smith, J.; Struhl, K. Current Protocols in Molecular Biology; John Wiley \& Sons, Inc.: New York, NY, USA, 1995.

54. Langmead, B.; Trapnell, C.; Pop, M.; Salzberg, S.L. Ultrafast and memory-efficient alignment of short DNA sequences to the human genome. Genome Biol. 2009, 10, R25. [CrossRef] [PubMed]

55. Luo, R.; Wong, T.; Zhu, J.; Liu, C.-M.; Zhu, X.; Wu, E.; Lee, L.-K.; Lin, H.; Zhu, W.; Cheung, D.W. SOAP3-dp: Fast, accurate and sensitive GPU-based short read aligner. PLoS ONE 2013, 8, e65632. [CrossRef] [PubMed]

56. Lagesen, K.; Hallin, P.; Rodland, E.A.; Staerfeldt, H.H.; Rognes, T.; Ussery, D.W. RNAmmer: Consistent and rapid annotation of ribosomal RNA genes. Nucleic Acids Res. 2007, 35, 3100-3108. [CrossRef] [PubMed]

57. Lowe, T.M.; Eddy, S.R. tRNAscan-SE: A program for improved detection of transfer RNA genes in genomic sequence. Nucleic Acids Res. 1997, 25, 955-964. [CrossRef] [PubMed]

58. Smit, A.; Hubley, R.; Green, P. Repeatmasker Open-4.0. Available online: http://www.Repeatmasker.Org (accessed on 30 November 2016).

59. Nawrocki, E.P.; Burge, S.W.; Bateman, A.; Daub, J.; Eberhardt, R.Y.; Eddy, S.R.; Floden, E.W.; Gardner, P.P.; Jones, T.A.; Tate, J.; et al. Rfam 12.0: Updates to the RNA families database. Nucleic Acids Res. 2015, 43, D130-D137. [CrossRef] [PubMed]

60. Nawrocki, E.P.; Eddy, S.R. Infernal 1.1: 100-fold faster RNA homology searches. Bioinformatics 2013, 29, 2933-2935. [CrossRef] [PubMed]

61. Lei, J.; Sun, Y. miR-prefer: An accurate, fast and easy-to-use plant miRNA prediction tool using small RNA-seq data. Bioinformatics 2014, 30, 2837-2839. [CrossRef] [PubMed]

62. Altschul, S.F.; Gish, W.; Miller, W.; Myers, E.W.; Lipman, D.J. Basic local alignment search tool. J. Mol. Biol. 1990, 215, 403-410. [CrossRef]

63. Kozomara, A.; Griffiths-Jones, S. miRBase: Annotating high confidence microRNAs using deep sequencing data. Nucleic Acids Res. 2014, 42, D68-D73. [CrossRef] [PubMed]

64. Anders, S.; Huber, W. Differential expression analysis for sequence count data. Genome Biol. 2010, 11, R106. [CrossRef] [PubMed]

65. Wang, L.; Feng, Z.; Wang, X.; Wang, X.; Zhang, X. Degseq: An R package for identifying differentially expressed genes from RNA-seq data. Bioinformatics 2010, 26, 136-138. [CrossRef] [PubMed]

66. Dai, X.; Zhao, P.X. psRNATarget: A plant small RNA target analysis server. Nucleic Acids Res. 2011, 39, W155-W159. [CrossRef] [PubMed]

67. Chen, H.-M.; Chen, L.-T.; Patel, K.; Li, Y.-H.; Baulcombe, D.C.; Wu, S.-H. 22-Nucleotide RNAs trigger secondary siRNA biogenesis in plants. Proc. Natl. Acad. Sci. USA 2010, 107, 15269-15274. [CrossRef] [PubMed]

(c) 2016 by the authors; licensee MDPI, Basel, Switzerland. This article is an open access article distributed under the terms and conditions of the Creative Commons Attribution (CC-BY) license (http://creativecommons.org/licenses/by/4.0/). 